\title{
Particles bed morphology under an oscillating stiff and flexible plates
}

\author{
Anna Prati ${ }^{1, *}$, Michele Larcher ${ }^{1, * *}$, James T. Jenkins ${ }^{2, * * *}$, and Luigi La Ragione ${ }^{3, * * * *}$ \\ ${ }^{1}$ Faculty of Science and Technology, Free University of Bozen-Bolzano, 39100 Bolzano, Italy \\ ${ }^{2}$ School of Civil and Environmental Engineering, Cornell University, Ithaca, New York 14853, USA \\ ${ }^{3}$ Department of Civil Engineering Sciences and Architecture, Polytechnic University of Bari, 70125 Bari, Italy
}

\begin{abstract}
We analyse the different morphologies induced by an oscillating plate above an erodible bed. We present some data describing how the shape and the stiffness of the plate affects the main features of the generated heap. We investigate several configurations with different geometries, frequencies and the amplitudes. Some preliminary results are available, in which the role of the flexibility of the plate is taken in account. Unlike the rigid plate in which a proper oscillation induces the formation of one heap, the morphology of the bed is now characterized by more than one heap due to a different pressure profile induced by a flexible plate.
\end{abstract}

\section{Introduction}

La Ragione et al. [1] have brought attention to a mechanism of failure for a fluid-saturated bed in an oscillated flow. They show that an oscillating plate in the water above a fluid-saturated particle bed induces pressure gradients that provoke particle motion and a formation of a heap. This is an example of failure in absence of mean shearing [2], in which the formation of a heap is associated with creeping that causes small rearrangements of the particles, likely driven by a gradient in pressure rather than shear stresses [3-5]. Here, we extend the experimental activity by employing plates with different dimensions and flexibility. In particular, we focus on the role of the stiffness of the plate and how this affects the morphology of the bed after failure occurs. Data for the present work have been collected using two different laser sheets, high speed cameras and a hydrophone to measure the pressure above the bed.

\section{Theory}

We briefly review the theory that supported the experimental activity in [1]. In Fig. 1a we sketch the essential elements of the experiment. As the plate oscillates with a given frequency $f$ and amplitude $A$, a vertical pore pressure gradient is induced in the bed, so when it is larger than the buoyant specific weight of the particles, a vertical motion occurs. This motion is different when the plate moves upwards or downwards, creating an irreversible feature in the bed, with a formation of a heap, that is evidence

\footnotetext{
*e-mail: anna.prati@natec.unibz.it

**e-mail: michele.larcher@unibz.it

***e-mail: jim.jenkins@cornell.edu

****e-mail: luigi.laragione@poliba.it
}

A video is available at https://doi.org/10.48448/7d2c-1f63 of expansion of the material. La Ragione et al. [1] find that the gradient in pressure is approximately equal to

$$
-\left.\frac{d p^{\prime}}{d y}\right|_{\max }=32 \rho A f^{2}
$$

which is balanced by the weight of the bed, to obtain

$$
\left(A f^{2}\right)_{\text {failure }}=\frac{\left(\rho_{s}-\rho\right)}{32 \rho} c g,
$$

in which $\rho_{s}$ is the particle density and $\rho$ is the water density. Experimental data support the predicted failure and indicate the role of the amplitude and frequency in failure, when a rigid plate oscillates. In the present work, we examine the role of the plate flexibility, so the theory should be employed accordingly. This will be part of a sequel; while, here, we report an initial set of experiments showing similarity and difference with the previous work.

\section{Experimental activity}

The experimental set up (displayed in Fig. 1a) consists of a transparent tank in which the particle bed is placed. Below the water free surface and above the bed surface a plate is placed and is oscillated by a Mini-shaker Type 4810, to which the plate is connected by means of a steel rod. The performance limits of this instrument (provided by Bruel and Kjaer) are defined by the maximum displacement (4 $\mathrm{mm}$ ) and maximum force (10 $\mathrm{N}$ or $7 \mathrm{~N}$, depending on frequency). The experimental procedure involved the use of two different laser sheets (as displayed in Fig. 1b), that allowed highlighting the profiles of the bedforms, and the use of two different high speed cameras to record the evolving phenomenon. The wavelength of the lasers are 532 $\mathrm{nm}$ (green) and $488 \mathrm{~nm}$ (red). Two laser sources of light, 

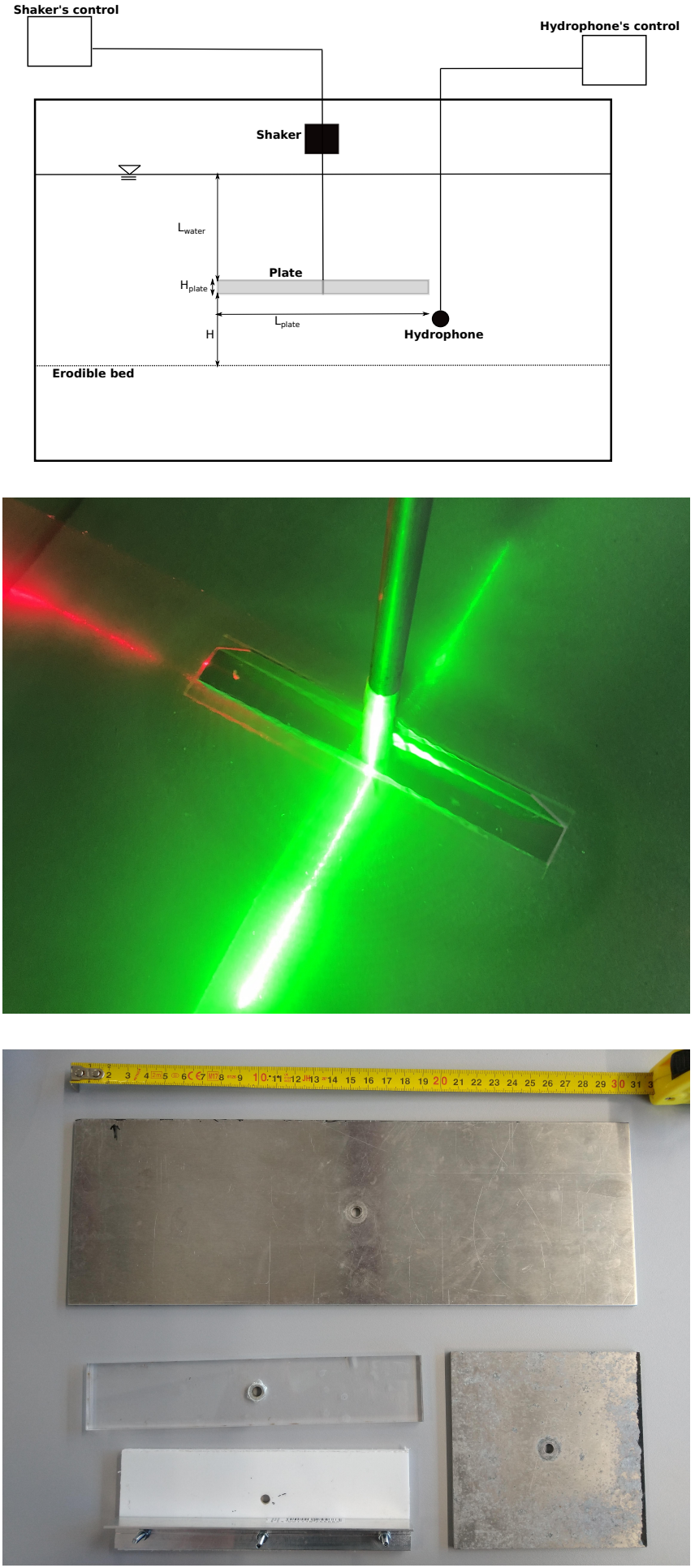

Fig. 1: The experimental set up. Top panel: sketch of the set up with the main physical distances involved in the experiments. Central panel: detail of the experimental set up with the two perpendicular laser sheets. Bottom panel: the plates used for this work. and two cameras, were necessary, because both the longitudinal and the transversal profiles of the bedforms have been investigated. It is worth pointing out that two different frame rates have been employed, depending on the purpose of the recordings: a high frame rate, between 250 and $600 \mathrm{fps}$, has been employed when the oscillation's amplitude of the plate was measured, while a low frame rate, between 0.1 and $10 \mathrm{fps}$, was employed to track the slow evolving bedform below the plate. To obtain the data exposed in the next subsections, images (in black and white) taken with the cameras have then been analysed by means of Matlab routines specific for image-processing purposes.

Oscillation amplitude of the plate. To evaluate the amplitude of the displacement induced on the plates by the accelerometer, we marked specific (small) circles on the thickness of the plates, tracked the positions of these dots and reconstructed the excursion of their displacements.

Heap profiles. The profiles of the heaps have been identified, in each frame, by exploiting the contrast between the brightness of the laser sheets and the darkness of the background. The laser enlightens the heap's edge, thus allowing to reconstruct its growth during the experiment.

The experimental analysis described in this work aims at comparing the modifications induced into a mobile bed by the vibration of different plates. Tab. 1 contains the geometric features of the plates used for the experiments and includes the shaker's oscillation amplitudes (measured in voltage and frequency) as well: by $A R$ and by $H_{p}$ we indicate, respectively, the aspect ratio and the thickness of the plate. All the plates used for this work are pictured in Fig. 1c and listed below:

1. rigid, square, metal plate $(l=10 \mathrm{~mm})$

2. flexible, rectang., metal plate $\left(l_{1}=10 \mathrm{~cm}, l_{2}=3 l_{1}\right)$

3. rigid, rectang., metal plate $\left(l_{1}=10 \mathrm{~cm}, l_{2}=3 l_{1}\right)$

4. flexible, rectang., plastic plate $\left(l_{1}=5 \mathrm{~cm}, l_{2}=4 l_{1}\right)$

5. rigid, rectang., plastic plate $\left(l_{1}=5 \mathrm{~cm}, l_{2}=4 l_{1}\right)$

6. flexible, rectang., plastic plate $\left(l_{1}=3.5 \mathrm{~cm}, l_{2}=5 l_{1}\right)$

7. rigid, rectang., plastic plate $\left(l_{1}=3.5 \mathrm{~cm}, l_{2}=5 l_{1}\right)$

\section{Results}

\subsection{The flexion data}

To better understand the results, the description of what we indicate as rigid and flexible plate is essential. This difference regards how the dynamic (and periodic) load generated by the shaker deforms the plates, and, specifically, how they are flexed and how their deformed line looks like during the stressing phase. In order to measure this flexion, we exploited the same dots drawn for the amplitude measurements. In the first plot of Fig. 2 the displacements of the dots on the $1 \times 1$ metal plate are plotted, while the 
Table 1: Experimental settings for each presented run.

\begin{tabular}{lllllll}
\hline Run & $\begin{array}{l}f \\
{[\mathrm{~Hz}]}\end{array}$ & $\begin{array}{l}\text { V } \\
{[\text { Volt }]}\end{array}$ & St./Fl. & Mat. & $A R$ & $\begin{array}{l}H_{p} \\
{[\mathrm{~mm}]}\end{array}$ \\
\hline 69 & 60 & 7 & stiff & met. & 1 & 3.2 \\
85 & 60 & 7 & flex & met. & 3 & 3.2 \\
145 & 60 & 7 & stiff & met. & 3 & 3.2 \\
163 & 60 & 7.5 & flex & plast. & 4 & 5 \\
164 & 60 & 7.5 & stiff & plast. & 4 & 5 \\
70 & 60 & 7 & flex & plast. & 3 & 10 \\
166 & 60 & 6.5 & stiff & plast. & 5 & 5 \\
\hline
\end{tabular}
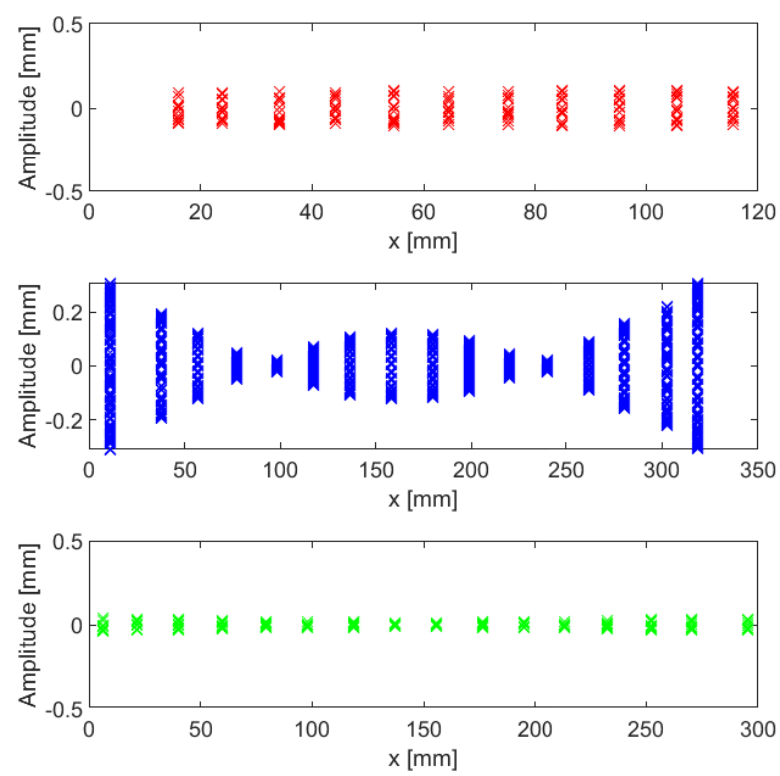

Fig. 2: Flexion data of three different plates used for the experiments: the rigid-square metal plate (at the top), the flexiblerectangular metal one (in the middle) and the rigid-rectangular metal one (at the bottom).

second plot of Fig. 2 displays the same type of data for the $1 \times 3$ metal plate. The difference between the two images clearly suggests that the first, square plate behaves as a rigid one, whereas the second, rectangular plate is flexible. To increase the stiffness of this latter plate we screwed a pair of very light, L-shaped, metal strips on the top of the plate and we repeated the flexion measurements, whose results are reported in the third plot of Fig. 2.

\subsection{The heap profiles}

The first result concerns the difference between the bedforms created. The different profiles mirror the different stress-patterns that the plates convey to the erodible bed. Specifically, our experiments proved that, while a rigid plate induces a single heap, a shaking, flexible plate promotes the growing of multiple heaps in the bed. In Fig. 3 we have subsequent images of the heap grown using the $1 \times 3$ plastic and flexible shaking plate; on the contrary,
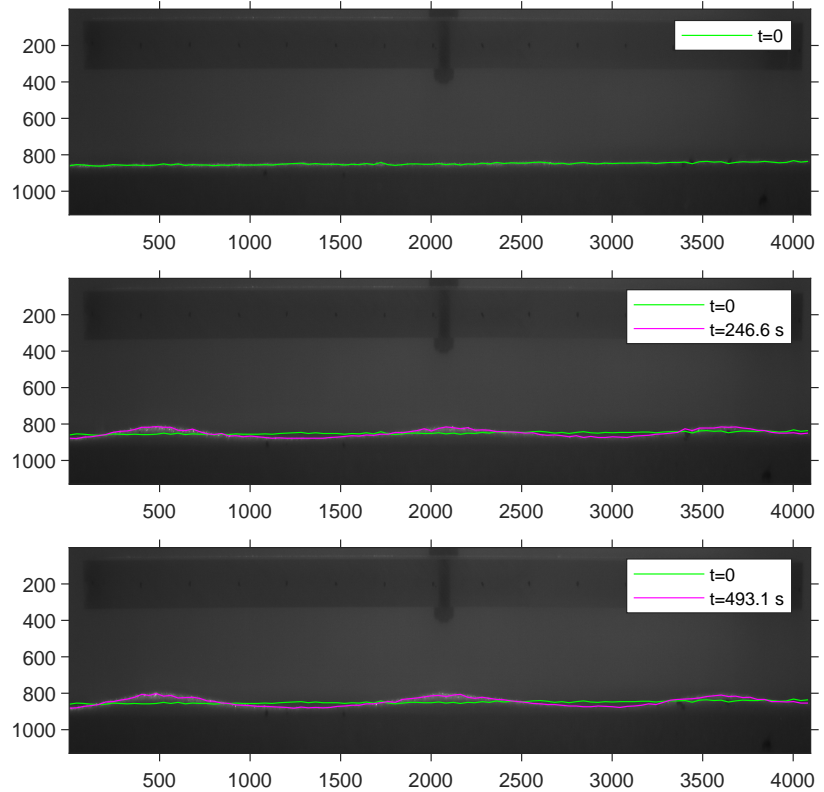

Fig. 3: Run n.70, both the axes are in pixel (where 1300 pixels correspond to $10 \mathrm{~cm}$ ).
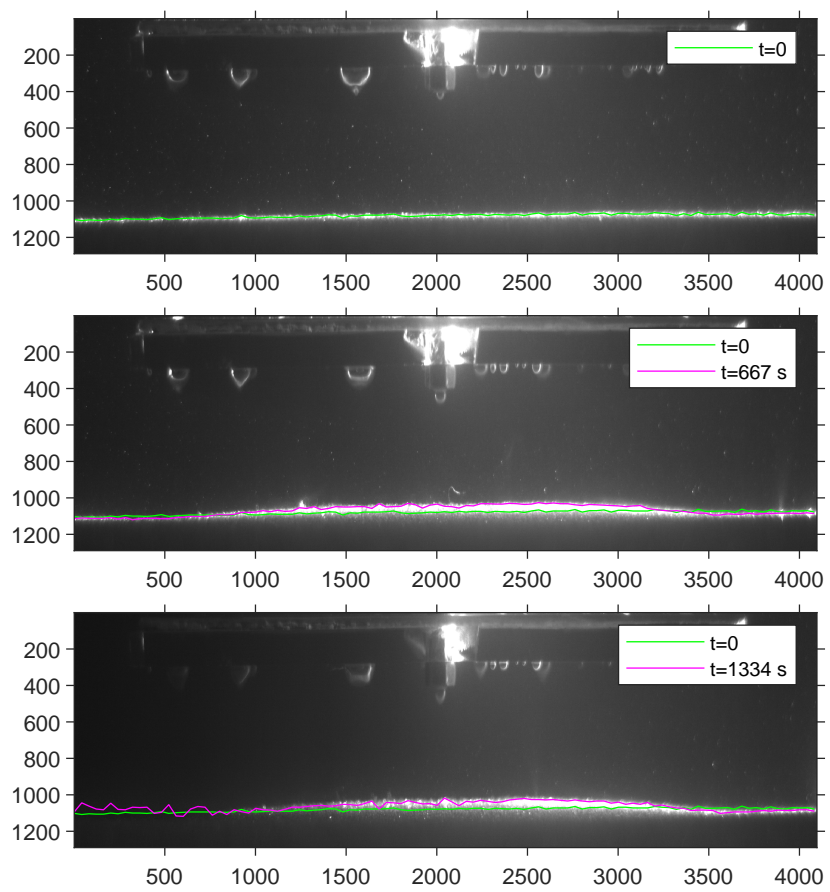

Fig. 4: Run n.166, both the axes are in pixel (where 1800 pixels correspond to $10 \mathrm{~cm}$ ).

Fig. 4 displays the heaps generated by the $1 \times 3$ plastic and rigid one. The parameters involved in the experiments are $f=60 \mathrm{~Hz}$ and $V=7 \mathrm{Volt}$, for the flexible plate, and $f=60 \mathrm{~Hz}$ and $V=6.5$ Volt, for the rigid plate. In Figs. $5 \mathrm{a}$ and $5 \mathrm{~b}$, we plot all the profiles (from the beginning to the end of the run) for the runs described above: the data are converted from pixel to millimetres. The parameters 
involved in the experiments are $f=60 \mathrm{~Hz}$ and $\mathrm{V}=7 \mathrm{Volt}$, for the flexible plate, and $f=60 \mathrm{~Hz}$ and $V=6.5 \mathrm{Volt}$, for the rigid plate.
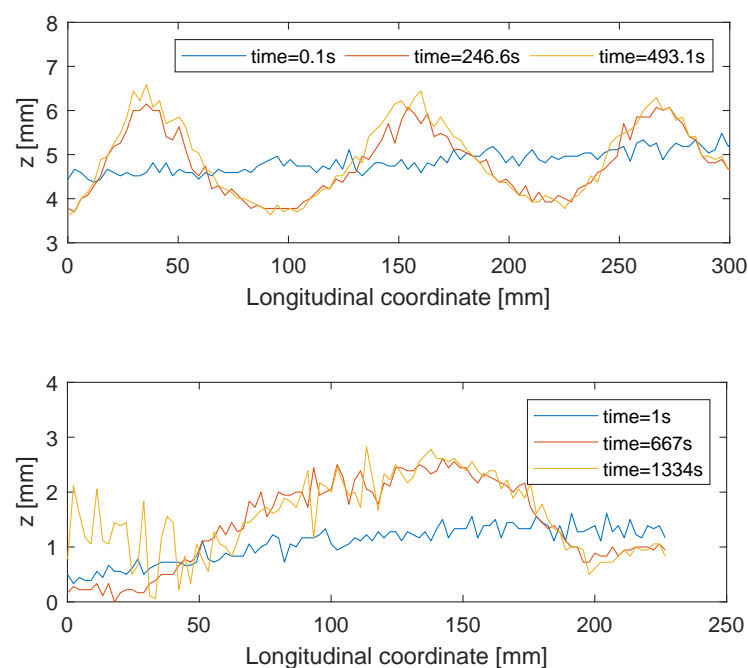

Fig. 5: Profiles at different times of run n.70 (above) and run n. 166 (below) without the background images (the conversion between pixel and $\mathrm{cm}$ is the same of the previous two figures).

\subsection{The pressure data}

We present now the pressure data acquired by slowly moving a very sensitive hydrophone below the entire length of the plates. As already explained in the previous sections, rigid and a flexible plates behave differently and, consistently, induce different pressure fields in the water (and on the bed). Fig. 6a displays the pressure data measured below two distinguished plates: the blue dots represent the pressure values induced by a flexible plate and the red dots represent the pressure values induced by a rigid one. For the sake of clarity, only half of the plates' length is shown: the longitudinal coordinate is null exactly below the center point of the plates and it is 0.5 below the external edge of the plates. Furthermore, for both the rigid and the flexible plate, the shaker was vibrating at $f=60 \mathrm{~Hz}$ and with an intensity of $V=7.5$ Volt. The two plates show behaviours and, specifically, the pressure envelope induced by the flexible plate (blue dots) exhibits a minimum (in its absolute value) at the very center of the plate. The flexible plate is responsible for the creation of two heaps and one of them is displayed in Fig. 6b. On the contrary the pressure profile induced by a rigid plate (red dots) clearly exhibits a maximum below the center point of the plate: such a plate causes indeed the growth of one single heap (as the one displayed, for instance, in the sequence of Fig. 4).

\section{Conclusions}

The experimental data have shown an interesting development of the previous research in which failure of a satu- rated particle bed has been achieved by means of gradient pressures induced by an oscillating rigid plate. Here we have investigated the role of the flexibility of the plate and the corresponding pressure over and inside the bed. We show the presence of multiple heaps over the bed surface instead of the single heap in case of rigid plate.
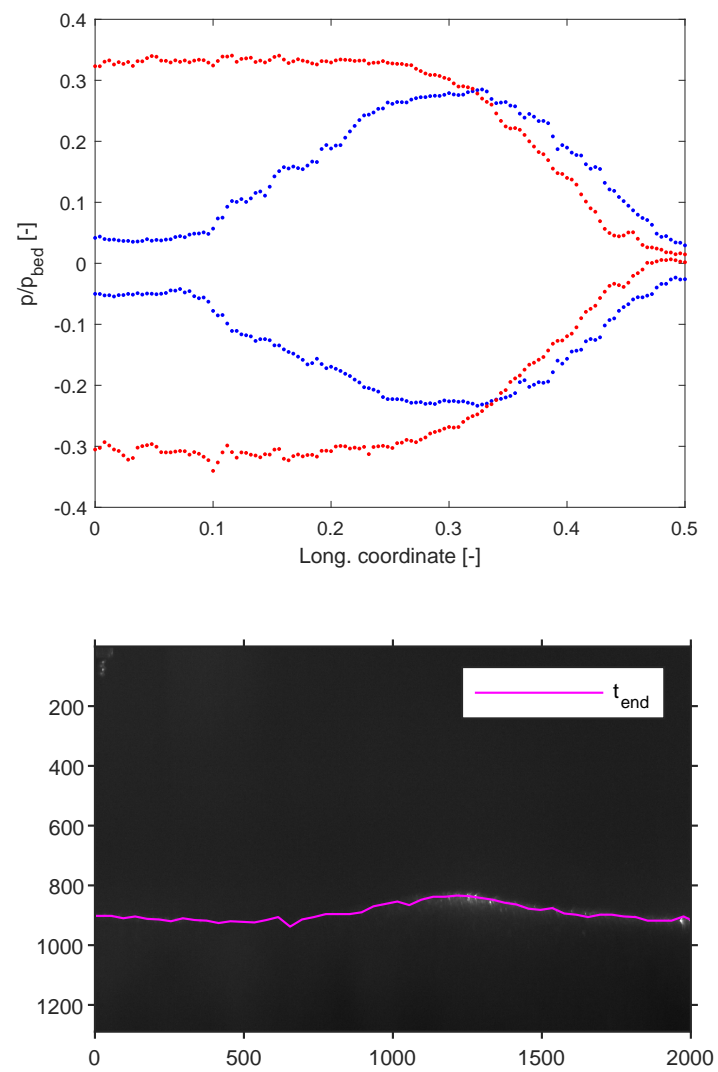

Fig. 6: Results for a flexible and rigid plate. Top panel: Pressure envelopes of minimum and maximum values for the flexible and the stiff plates (run n. 163 and 164). Bottom panel: Heap profile at the end of run n.163 where the flexible plate was used (where 1800 pixels correspond to $10 \mathrm{~cm}$ ).

\section{References}

[1] L. La Ragione, K. Laurent, J.T. Jenkins, G.P. Beweley, Phys. Rev. Lett. 123, 058501 (2019)

[2] B.A. Johnson, E.A. Cowen, J. Fluid Mech. 894 (2020)

[3] M. Houssais, C.P. Ortiz, D.J. Durian, D.J. Jerolmack, Nat. Commun. 6, 6527 (2015)

[4] P. Marchal, C. Hanotin, L.J. Michot, S. de Richter, Phys. Rev. E. 88, 012207 (2013)

[5] C. Hanotin, S. de Richter, P. Marchal, L.J. Michot, C. Baravian, Phys. Rev. Lett. 19, 198301 (2012) 